\title{
Positive Solutions for Systems of Fourth Order Two-Point Boundary Value Problems with Parameter
}

\author{
Noureddine Bouteraa ${ }^{1}$, Slimane Benaicha ${ }^{1}$ and Habib Djourdem ${ }^{1^{*}}$ \\ ${ }^{1}$ Laboratory of Fundamental and Applied Mathematics of Oran (LMFAO), University of Oran1, Ahmed Benbella. Algeria \\ ${ }^{*}$ Corresponding author
}

\section{Article Info}

Keywords: Fixed-point theorem, Green's function, Positive solutions, System of differential equations, Two-point boundary value problem. 2010 AMS: 34B10,34B18.

Received: 10 June 2018

Accepted: 1 January 2019

Available online: 20 April 2019

\begin{abstract}
This paper deals with the existence of positive solutions for a system of nonlinear singular fourth-order differential equations with a parameter $\lambda$ subject two-point boundary conditions. Our analysis relies on the Krasnoselskii fixed point theorem and under suitable conditions, we derive explicit eigenvalue intervals of $\lambda$ for the existence of at least one positive solution for the system.
\end{abstract}

\section{Introduction}

We are concerned with determining intervals of the parameter $\lambda$ for which there exist positive solutions for the following boundary value problem of nonlinear differential system BVPs

$$
\begin{cases}u^{(4)}(t)+\lambda a(t) f(v(t))=0, & 0<t<1 \\ v^{(4)}(t)+\lambda b(t) g(u(t))=0, & 0<t<1\end{cases}
$$

subject to two-point boundary conditions.

$$
\left\{\begin{array}{l}
u(0)=0, u^{\prime}(0)=0, u^{\prime \prime}(1)=0, u^{\prime \prime \prime}(1)=0 \\
v(0)=0, v^{\prime}(0)=0, v^{\prime \prime}(1)=0, v^{\prime \prime \prime}(1)=0
\end{array}\right.
$$

The theory of multi-point boundary value problems for ordinary differential equations arises in different areas of applied mathematics and physics. For example, the vibrations of a guy wire of uniform cross-section and composed of $\mathrm{N}$ parts of different densities that can be set up as a multi-point boundary value problem. Many problems in the theory of elastic stability can be handled as multi-point boundary value problems too. Higher-order boundary value problems occur in the study of fluid dynamics, astrophysics, hydrodynamic, hydromagnetic stability and astronomy, be a mandlong wave theory, induction motors, engineering and applied physics. The boundary value problems of higher-order have been examined due to their mathematical importance and applications in different areas of applied sciences. In particular, third-order, fourth-order and nth order were considered, see [1] - [14] and the references therein.

Fourth-order ordinary differential equations are models for bending or deformation of elastic beams, and therefore have important applications in mechanics, engenieering and physical sciences; see [15] - [19]. Many authors have studied the beam equation under various boundary conditions and by different approaches. For example, Bouteraa et al. [20,21], studied the existence and nonexistence of positive solutions of two types boundary value problem for a nonlinear fourth-order differential equation

$$
\left\{\begin{array}{lc}
u^{(4)}(t)=\lambda f(t, u(t)), & t \in(0,1) \\
u(0)=u^{\prime}(0)=u^{\prime}(1)=u^{\prime \prime \prime}(1)+\psi(u(1))=0
\end{array}\right.
$$

Email addresses and ORCID numbers: bouteraa-27@ hotmail.fr, 0000-0002-8772-1315 (N. Bouteraa), slimanebenaicha@yahoo.fr, 0000-0002-8953-8709 (S. Benaicha), djourdem.habib7@gmail.com, 0000-0002-7992-581X (H. Djourdem) 
and

$$
\left\{\begin{array}{c}
u^{(4)}(t)-\rho^{4} u(t)=f(t, u(t)), t \in[0, \omega] \\
u^{(i)}(0)=u^{(i)}(\omega), \quad i \in\{0,1,2,3\}
\end{array}\right.
$$

where $\lambda>0, \rho \in \mathbb{R}^{+}, f \in C([0, \omega] \times \mathbb{R}, \mathbb{R})$ and $\psi \in C([0, \infty),[0, \infty))$.

By applying iterative method, Djourdem et al. [22] obtained the existence of monotone positive solution of the following nonlinear BVP

$$
\begin{gathered}
u^{(4)}(t)=f(t, u(t)), \quad t \in[0,1], \\
u^{\prime}(0)=u^{\prime \prime}(0)=u(1)=0, u^{\prime \prime \prime}(\eta)+\alpha u(0)=0,
\end{gathered}
$$

where $f \in C([0,1] \times[0,+\infty),[0,+\infty)), \alpha \in[0,6)$ and $\eta \in\left[\frac{2}{3}, 1\right)$.

However, there are few works that deal with multi-point boundary value problem for a coupled systems of nonlinear differential equations, see [23] - [25]. Motivated by the above montioned works and works of coupled systems of nonlinear differential equations, we discuss the existence of positive solutions of BVPs (1.1)-(1.2). Ours analysis relies on the Guo-Krasnoselskii's fixed point theorem for operators leaving a Banach space cone invariant [26]. A Green function play a fundamental role in defining an appropriate operator on a suitable cone. The aim of this paper is to etablish some simple criteria for the existence of single positive solutions of the BVPs (1.1)-(1.2) in explicit intervals for $\lambda$. This paper is organized as follows. In section 2, we present some preliminaries and lemmas that will be used to prove our main results. In section 3, we discuss the existence of single positive solution of BVPs (1.1)-(1.2), and an example explain our conditions are applicable.

\section{Preliminaries}

Let $B=C([0,1], \mathbb{R})$ be a Banach space endowed with usual supermum norm, and $B^{+}=C\left([0,1], \mathbb{R}^{+}\right)$.

Lemma 2.1. Let $y(\cdot) \in C[0,1]$. If $u \in C^{4}[0,1]$, then the $B V P$

$$
\left\{\begin{array}{lr}
u^{(4)}(t)=y(t), & 0 \leq t \leq 1 \\
u(0)=u^{\prime}(0)=u^{\prime \prime}(1)= & u^{\prime \prime \prime}(1)=0
\end{array}\right.
$$

has a unique solution

$$
u(t)=\int_{0}^{1} H(t, s) y(s) d s
$$

where

$$
H(t, s)= \begin{cases}\frac{1}{6} t^{2}(3 s-t), & 0 \leq t \leq s \leq 1 \\ \frac{1}{6} s^{2}(3 t-s), & 0 \leq s \leq t \leq 1\end{cases}
$$

Proof. The derivatives of the function $H$ with respect to $t$ is

$$
\frac{\partial}{\partial t} H(t, s)=\left\{\begin{array}{c}
\frac{1}{2} s^{2}-\frac{1}{2}(s-t)^{2}, 0 \leq t \leq s \leq 1 \\
\frac{1}{2} s^{2}, \quad 0 \leq s \leq t \leq 1
\end{array}\right.
$$

Since the derivative of the function $H$ with respect to $t$ is nonnegative for all $t \in[0,1], H$ is nondecreasing function of $t$ that attaints its maximum when $t=1$. Then

$$
\max _{0 \leq t \leq 1} H(t, s)=H(1, s)=\frac{1}{2} s^{2}-\frac{1}{6} s^{3}=\psi(s)
$$

Lemma 2.2. Let $y(\cdot) \in B^{+}$. Then, the unique solution $u(t)$ of BVPs $(1.1)-(1.2)$ is nonnegative and satisfies

$$
\min _{t \in[\theta, 1]} u(t) \geq \frac{2 \theta^{3}}{3}\|u\|
$$

where $\theta \in(0,1)$.

Proof. Let $y(\cdot) \in B^{+}$, then from $H(t, s) \geq 0$, we know $u \in B^{+}$. Set $u\left(t_{0}\right)=\|u\|, t_{0} \in(0,1]$. we first prove that

$$
\frac{H(t, s)}{H\left(t_{0}, s\right)} \geq \frac{2}{3} t^{3}, \quad t, t_{0}, s \in(0,1] .
$$

In fact, we can consider four cases:

(1) if $0<t, t_{0} \leq s \leq 1$, then

$$
\frac{H(t, s)}{H\left(t_{0}, s\right)}=\frac{t^{2}(3 s-t)}{t_{0}^{2}\left(3 s-t_{0}\right)} \geq \frac{t^{2}(2 s)}{3 t_{0}-t_{0}} \geq \frac{t^{2}(2 s)}{3} \geq \frac{t^{2}(2 t)}{3}=\frac{2 t^{3}}{3}
$$


(2) if $0 \leq t \leq s \leq t_{0} \leq 1$, then

$$
\frac{H(t, s)}{H\left(t_{0}, s\right)}=\frac{t^{2}(3 s-t)}{s^{2}\left(3 t_{0}-s\right)} \geq \frac{t^{2}(3 s-t)}{3 t_{0}-s} \geq \frac{t^{2}(3 s-s)}{3 t_{0}} \geq \frac{t^{2}(2 s)}{3} \geq \frac{t^{2}(2 t)}{3} \geq \frac{2 t^{3}}{3},
$$

(3) if $0<s \leq t, t_{0} \leq 1$, then

$$
\frac{H(t, s)}{H\left(t_{0}, s\right)}=\frac{s^{2}(3 t-s)}{s^{2}\left(3 t_{0}-s\right)}=\frac{3 t-s}{3 t_{0}-s} \geq \frac{3 t-s}{3 t_{0}} \geq \frac{3 t-s}{3} \geq \frac{2 t+t-s}{3} \geq \frac{2 t}{3} \geq \frac{2 t^{3}}{3},
$$

(4) if $0 \leq t_{0} \leq s \leq t \leq 1$, then

$$
\frac{H(t, s)}{H\left(t_{0}, s\right)}=\frac{s^{2}(3 t-s)}{t_{0}^{2}\left(3 s-t_{0}\right)} \geq \frac{t_{0}^{2}(3 t-s)}{t_{0}^{2}\left(3 t-t_{0}\right)} \geq \frac{3 t-s}{3 t} \geq \frac{3 t-t}{3 t} \geq \frac{2 t}{3} \geq \frac{2 t^{3}}{3}
$$

Therefore, for $t \in[\theta, 1]$, we obtain

$$
\begin{aligned}
u(t) & =\int_{0}^{1} H(t, s) y(s) d s \\
& =\int_{0}^{1} \frac{H(t, s)}{H\left(t_{0}, s\right)} H\left(t_{0}, s\right) y(s) d s \\
& \geq \int_{0}^{1} \frac{2 t^{3}}{3} H\left(t_{0}, s\right) y(s) d s=\frac{2 t^{3}}{3} u\left(t_{0}\right) \geq \frac{2 \theta^{3}}{3}\|u\| .
\end{aligned}
$$

The proof is complete.

If we let

$$
K=\left\{u \in B: u(t) \geq 0 \text { on }[0,1] \text { and } \min _{t \in[\theta, 1]} u(t) \geq \frac{2 \theta^{3}}{3}\|u\|\right\}
$$

then it is easy to see that $K$ a cone in $B$.

We not that the BVPs $(1.1)-(1.2)$ has a solutin $(u(t), v(t))$ if, and only if

$$
u(t)=\lambda \int_{0}^{1} H(t, s) a(s) f\left(\lambda \int_{0}^{1} H(s, v) b(v) g(u(v)) d v\right) d s, \quad t \in[0,1],
$$

and

$$
v(t)=\lambda \int_{0}^{1} H(t, s) b(s) g(u(s)) d s, \quad t \in[0,1]
$$

Now, we define an integral operator $Q_{\lambda}: B^{+} \rightarrow B$ by

$$
\left(Q_{\lambda} u\right)(t)=\lambda \int_{0}^{1} H(t, s) a(s) f\left(\lambda \int_{0}^{1} H(s, v) b(v) g(u(v)) d v\right) d s, \quad u \in K .
$$

We adopt the following hypothesies:

$\left(H_{1}\right) a, b \in C((0,1),[0, \infty))$ and each does not vanish identically on any subinterval.

$\left(H_{2}\right) f, g \in C([0, \infty),[0, \infty))$.

$\left(H_{3}\right)$ All of $f_{0}=\lim _{x \rightarrow 0^{+}} \frac{f(x)}{x}, g_{0}=\lim _{x \rightarrow 0^{+}} \frac{g(x)}{x}, f_{\infty}=\lim _{x \rightarrow \infty} \frac{f(x)}{x}, g_{\infty}=\lim _{x \rightarrow \infty} \frac{g(x)}{x}$ exist as real numbers.

$\left(H_{4}\right) g(0)=0$ and $f$ is increasing function.

Lemma 2.3. Let $\lambda>0$ and $K$ be the cone defined above.

(i) If $u \in B^{+}$and $v:[0,1] \rightarrow[0, \infty)$ is defined by (2.2), then $v \in K$.

(ii) If $Q_{\lambda}$ is the integral operator defined by (2.3), then $Q_{\lambda}(K) \subset K$.

(iii) Suppose $\left(H_{1}\right)$ and $\left(H_{2}\right)$ hold. Then $Q_{\lambda}: K \rightarrow B$ is completely continuous. 
Proof. Let $u \in B^{+}$and $v$ be defined by (2.2).

(i) By the nonnegativity of $H, b$ and $g$ it follows that $v(t) \geq 0, t \in[0,1]$. In view of $\left(H_{1}\right),\left(H_{2}\right)$, we obtain

$$
\int_{0}^{1} H(s, v) b(v) g(u(v)) d v \geq \int_{0}^{1} \min _{s \in[\theta, 1]} H(s, v) b(v) g(u(v)) d v
$$

from which, we take

$$
\min _{s \in[\theta, 1]} \int_{0}^{1} H(s, v) b(v) g(u(v)) d v \geq \int_{0}^{1} \min _{s \in[\theta, 1]} H(s, v) b(v) g(u(v)) d v
$$

Consequently, employing (2.2), we obtain

$$
\begin{aligned}
\int_{0}^{1} H(s, v) b(v) g(u(v)) d v & \geq \int_{0}^{1} \min _{s \in[\theta, 1]} H(s, v) b(v) g(u(v)) d v \\
& \geq \frac{2 \theta^{3}}{3} \int_{0}^{1} H\left(s_{0}, v\right) b(v) g(u(v)) d v \\
& \geq \frac{2 \theta^{3}}{3} v\left(s_{0}\right), s_{0} \in(0,1] \\
& \geq \frac{2 \theta^{3}}{3}\|v\| .
\end{aligned}
$$

Therefore

$$
\min _{0<s<1} v(s) \geq \frac{2 \theta^{3}}{3}\|v\|
$$

Which give that $v \in K$.

(ii) Obviously, for $v \in K, Q_{\lambda}(u) \in B^{+}$. For $t \in[0,1]$, we have

$$
\begin{aligned}
\left\|Q_{\lambda} u(t)\right\| & =\max _{0 \leq t \leq 1} \lambda \int_{0}^{1} H(t, s) a(s) f(v(s)) d s \\
& \leq \lambda \int_{0}^{1} H(1, s) a(s) f(v(s)) d s
\end{aligned}
$$

and

$$
\begin{aligned}
Q_{\lambda} u(t) & =\lambda \int_{0}^{1} H(t, s) a(s) f(v(s)) d s \\
& =\lambda \int_{0}^{1} \frac{H(t, s)}{H(1, s)} H(1, s) a(s) f(v(s)) d s \\
& \geq \frac{2 \theta^{3}}{3} \lambda \int_{0}^{1} H(1, s) a(s) f(v(s)) d s \\
& \geq \frac{2}{3} \theta^{3}\left\|Q_{\lambda} u(t)\right\| .
\end{aligned}
$$

Which give that $Q_{\lambda} u \in K$. Therefore $Q_{\lambda}: K \rightarrow K$.

(iii) It is not difficult to show that the operator $Q_{\lambda}: K \rightarrow B$ is completely continuous.

Our analysis relies on the following Krasnoselskii's fixed point theorem of cone expansion-compression type.

Theorem 2.4. (See [26]) Let $E$ be a Banach space and $K \subset E$ be a cone in E. Assume $\Omega_{1}$ and $\Omega_{2}$ are open subset of $E$ with $0 \in \Omega_{1}$ and $\overline{\Omega_{1}} \subset \Omega_{2}, Q: K \cap\left(\bar{\Omega}_{2} \backslash \Omega_{1}\right) \rightarrow K$ be a completely continuous operator such that

(i) $\|Q u\| \leq\|u\|$, for all $u \in K \cap \partial \Omega_{1}$ and $\|Q u\| \geq\|u\|, \forall u \in K \cap \partial \Omega_{2}$, or

(ii) $\|Q u\| \geq\|u\|, \forall u \in K \cap \partial \Omega_{1}$ and $\|Q u\| \leq\|u\|, \forall u \in K \cap \partial \Omega_{2}$

Then $Q$ has a fixed point in $K \cap\left(\bar{\Omega}_{2} \backslash \Omega_{1}\right)$. 
Throughout this paper, we shall use the following notations:

$$
\begin{gathered}
L_{1}=\max \left\{\left[\left(\frac{2 \theta^{3}}{3}\right)^{2} \int_{\theta}^{1} \psi(v) a(v) f_{\infty} d v\right]^{-1},\left[\left(\frac{2 \theta^{3}}{3}\right)^{2} \int_{\theta}^{1} \psi(v) a(v) g_{\infty} d v\right]^{-1}\right\}, \\
L_{2}=\min \left\{\left[\int_{0}^{1} \psi(v) a(v) f_{0} d v\right]^{-1},\left[\int_{0}^{1} \psi(v) b(v) g_{0} d v\right]^{-1}\right\}, \\
L_{3}=\max \left\{\left[\left(\frac{2 \theta^{3}}{3}\right)^{2} \int_{\theta}^{1} \psi(v) a(v) f_{0} d v\right]^{-1},\left[\left(\frac{2 \theta^{3}}{3}\right)^{2} \int_{\theta}^{1} \psi(v) a(v) g_{0} d v\right]^{-1}\right\}
\end{gathered}
$$

and

$$
L_{4}=\min \left\{\left[\int_{0}^{1} \psi(v) a(v) f_{\infty} d v\right]^{-1},\left[\int_{0}^{1} \psi(v) b(v) g_{\infty} d v\right]^{-1}\right\}
$$

\section{Main results}

This section deals with the existence of at least one positive solution for BVPs (1.1)-(1.2). Our analysis relies on the Krasnoselskii fixed point theorem Theorem 2.4.

Theorem 3.1. Under assumptions $\left(H_{1}\right),\left(H_{2}\right)$ and $\left(H_{3}\right)$, the BVPs (1.1)-(1.2) has a non-negative solution $(u, v)$ for any $\lambda$ satisfying $L_{1}<\lambda<L_{2}$.

Proof. Let $L_{1}<\lambda<L_{2}$ and choose $\varepsilon>0$ such that

$$
\max \left\{\left[\left(\frac{2 \theta^{3}}{3}\right)^{2} \int_{\theta}^{1} \psi(v) a(v)\left(f_{\infty}-\varepsilon\right) d v\right]^{-1},\left[\left(\frac{2 \theta^{3}}{3}\right)^{2} \int_{\theta}^{1} \psi(v) a(v)\left(g_{\infty}-\varepsilon\right) d v\right]^{-1}\right\} \leq \lambda
$$

and

$$
\lambda \leq \min \left\{\left[\int_{0}^{1} \psi(v) a(v)\left(f_{0}+\varepsilon\right) d v\right]^{-1},\left[\int_{0}^{1} \psi(v) b(v)\left(g_{0}+\varepsilon\right) d v\right]^{-1}\right\}
$$

From the definitions of $f_{0}$ and $g_{0}$ there exists an $R_{1}>0$ such that

$$
f(u) \leq\left(f_{0}+\varepsilon\right) u, 0<u \leq R_{1},
$$

and

$$
g(u) \leq\left(g_{0}+\varepsilon\right) u, 0<u \leq R_{1} .
$$

Let $u \in K$ with $\|u\|=R_{1}$. From (2.1) and choice of $\varepsilon$, we have

$$
\begin{aligned}
\lambda \int_{0}^{1} H(t, s) b(v) g(u(v)) & \leq \lambda \int_{0}^{1} \psi(v) b(v) g(u(v)) d v \\
& \leq \lambda \int_{0}^{1} \psi(v) b(v)\left(g_{0}+\varepsilon\right) u(v) d v \\
& \leq\|u\| \lambda \int_{0}^{1} \psi(v) b(v)\left(g_{0}+\varepsilon\right) d v \\
& \leq R_{1}=\|u\| .
\end{aligned}
$$


Consequently, from (2.1) and choice of $\varepsilon$, we obtain

$$
\begin{aligned}
& Q_{\lambda} u(t)=\lambda \int_{0}^{1} H(t, s) a(s) f\left(\lambda \int_{0}^{1} H(s, v) b(v) g(u(v)) d v\right) d s \\
& \leq \lambda \int_{0}^{1} \psi(s) a(s) f\left(\lambda \int_{0}^{1} H(s, v) b(v) g(u(v)) d v\right) d s \\
& \leq \lambda \int_{0}^{1} \psi(s) a(s)\left(f_{0}+\varepsilon\right)\left[\lambda \int_{0}^{1} H(s, v) b(v) g(u(v)) d v\right] d s \\
& \leq \lambda \int_{0}^{1} \psi(s) a(s)\left(f_{0}+\varepsilon\right) R_{1} d s \\
& \leq R_{1}=\|u\| .
\end{aligned}
$$

So, $\|T u\| \leq\|u\|$. If we set $\Omega_{1}=\left\{u \in B:\|u\|<R_{1}\right\}$, then

$$
\left\|Q_{\lambda} u\right\| \leq\|u\|, \text { for } u \in K \cap \partial \Omega_{1} .
$$

Considering the definitions of $f_{\infty}$ and $g_{\infty}$ there exists an $\bar{R}_{2}>0$ such that

$$
f(u) \geq\left(f_{\infty}-\varepsilon\right) u, \quad u \geq \bar{R}_{2},
$$

and

$$
g(u) \geq\left(g_{\infty}-\varepsilon\right) u, \quad u \geq \bar{R}_{2}
$$

Let $u \in K$ and $R_{2}=\max \left\{2 R_{1}, \frac{3 \bar{R}_{2}}{2 \theta^{3}}\right\}$ with $\|u\|=R_{2}$, then

$$
\min _{s \in[\theta, 1]} u(s) \geq \frac{2}{3} \theta^{3}\|u\| \geq \bar{R}_{2}
$$

Therefore, from (2.2) and choice of $\varepsilon$, we have

$$
\begin{aligned}
\lambda \int_{0}^{1} H(t, s) b(v) g(u(v)) d v & \geq \frac{2 \theta^{3}}{3} \lambda \int_{0}^{1} H(1, v) b(v) g(u(v)) d v \\
& \geq \frac{2 \theta^{3}}{3} \lambda \int_{\theta}^{1} \psi(v) b(v)\left(g_{\infty}-\varepsilon\right) u(v) d v \\
& \geq\|u\|\left(\frac{2 \theta^{3}}{3}\right)^{2} \lambda \int_{\theta}^{1} \psi(v) b(v)\left(g_{\infty}-\varepsilon\right) d v \\
& \geq R_{2}=\|u\| .
\end{aligned}
$$

Consequently, from (2.2), we obtain

$$
\begin{aligned}
Q_{\lambda} u(t) & \geq \frac{2 \theta}{3} \lambda \int_{\theta}^{1} \psi(s) a(s) f\left(\lambda \int_{\theta}^{1} H(s, v) b(v) g(u(v)) d v\right) d s \\
& \geq \frac{2 \theta^{3}}{3} \lambda \int_{\theta}^{1} \psi(s) a(s)\left(f_{\infty}-\varepsilon\right)\left[\lambda \int_{\theta}^{1} H(s, v) b(v) g(u(v)) d v\right] d s \\
& \geq \lambda \gamma \int_{\theta}^{1} \psi(s) a(s)\left(f_{\infty}-\varepsilon\right) R_{2} d s
\end{aligned}
$$




$$
\begin{aligned}
& \geq \lambda \gamma^{2} \int_{\theta}^{1} \psi(s) a(s)\left(f_{\infty}-\varepsilon\right) R_{2} d s \\
& \geq R_{2}=\|u\| .
\end{aligned}
$$

So, $\left\|Q_{\lambda} u\right\| \geq\|u\|$. If we set $\Omega_{2}=\left\{u \in B:\|u\|<R_{2}\right\}$, then

$$
\left\|Q_{\lambda} u\right\| \geq\|u\|, \text { for } u \in K \cap \partial \Omega_{2} .
$$

From of part (ii) of Theorem 2.4 to (3.1)and (3.1), the operator $Q_{\lambda}$ has a fixed point $u^{*} \in K \cap\left(\bar{\Omega}_{2} / \Omega_{1}\right)$. As such and with $v$ defined by

$$
v(t)=\lambda \int_{0}^{1} H(t, s) b(s) g(u(s)) d s .
$$

This means that the BVPs (1.1)-(1.2) has a nonnegative solution $(u, v)$ for the given $\lambda$. The proof is complete.

Theorem 3.2. Under assumptions $\left(H_{1}\right),\left(H_{2}\right),\left(H_{3}\right)$ and $\left(H_{4}\right)$, the BVPs (1.1)-(1.2) has a non-negative solution $(u, v)$ for any $\lambda$ satisfying $L_{3}<\lambda<L_{4}$.

Proof. Let $L_{3}<\lambda<L_{4}$ and choose $\varepsilon>0$ such that

$$
\max \left\{\left[\left(\frac{2 \theta^{3}}{3}\right)^{2} \int_{\theta}^{1} \psi(v) a(v)\left(f_{0}-\varepsilon\right) d v\right]^{-1},\left[\left(\frac{2 \theta^{3}}{3}\right)^{2} \int_{\theta}^{1} \psi(v) a(v)\left(g_{0}-\varepsilon\right) d v\right]^{-1}\right\} \leq \lambda,
$$

and

$$
\lambda \leq \min \left\{\left[\int_{0}^{1} \psi(v) a(v)\left(f_{\infty}+\varepsilon\right) d v\right]^{-1},\left[\int_{0}^{1} \psi(v) b(v)\left(g_{\infty}+\varepsilon\right) d v\right]^{-1}\right\} .
$$

From the definitions of $f_{0}$ and $g_{0}$ there exists an $R_{1}>0$ such that

$$
f(u) \geq\left(f_{0}-\varepsilon\right) u, \quad 0<u \leq R_{1},
$$

and

$$
g(u) \geq\left(g_{0}-\varepsilon\right) u, \quad 0<u \leq R_{1} .
$$

Now $g(0)=0$ and so there exists $0<R_{2}<R_{1}$ such that

$$
\lambda g(u) \leq \frac{R_{1}}{\int_{0}^{1} \psi(v) b(v) d v}, \quad 0 \leq u \leq R_{2} .
$$

Let $u \in K$ with $\|u\|=R_{2}$. Then

$$
\begin{aligned}
\lambda \int_{0}^{1} H(t, s) b(v) g(u(v)) \leq & \lambda \int_{0}^{1} \psi(v) b(v) g(u(v)) d v \\
& \leq \frac{\int_{0}^{1} \psi(v) b(v) R_{1} d v}{\int_{0}^{1} \psi(v) b(v) d v} \\
& \leq R_{1}=\|u\| .
\end{aligned}
$$

Therefore, by (2.2), we obtain

$$
\begin{gathered}
Q_{\lambda} u(t)=\lambda \int_{0}^{1} H(t, s) a(s) f\left(\lambda \int_{0}^{1} H(s, v) b(v) g(u(v)) d v\right) d s \\
\geq \frac{2 \theta^{3}}{3} \lambda \int_{\theta}^{1} \psi(s) a(s) f\left(\frac{2 \theta^{3}}{3} \lambda \int_{\theta}^{1} \psi(v) b(v) g(u(v)) d v\right) d s \\
\geq \frac{2 \theta^{3}}{3} \lambda \int_{\theta}^{1} \psi(s) a(s)\left(f_{0}-\varepsilon\right)\left[\left(\frac{2 \theta^{3}}{3}\right)^{2} \lambda \int_{\theta}^{1} \psi(v) b(v)\left(g_{0}-\varepsilon\right)\|u\| d v\right] d s
\end{gathered}
$$




$$
\begin{aligned}
& \geq\|u\| \frac{2 \theta^{3}}{3} \lambda \int_{\theta}^{1} \psi(v) a(v)\left(f_{0}-\varepsilon\right) d v \\
& \geq\|u\|\left(\frac{2 \theta^{3}}{3}\right)^{2} \lambda \int_{\theta}^{1} \psi(v) a(v)\left(f_{0}-\varepsilon\right) d v \\
& \geq\|u\| .
\end{aligned}
$$

So, $\left\|Q_{\lambda} u\right\| \geq\|u\|$. If we set $\Omega_{1}=\left\{u \in B:\|u\|<R_{2}\right\}$, then

$$
\left\|Q_{\lambda} u\right\| \geq\|u\|, \quad u \in K \cap\left(\bar{\Omega}_{2} \backslash \Omega_{1}\right) .
$$

Considering the definitions of $f_{\infty}$ and $g_{\infty}$ there exists $\bar{R}_{1}>0$ such that

$$
f(u) \leq\left(f_{\infty}+\varepsilon\right) u, \quad u \geq \bar{R}_{1},
$$

and

$$
g(u) \leq\left(g_{\infty}+\varepsilon\right) u, \quad u \geq \bar{R}_{1} .
$$

We consider two cases: $g$ is bounded or $g$ is unbounded.

Case $(i)$. Assume that $g$ is bounded, say $g(x) \leq N, \quad N>0$ for all $0<x<\infty$. Then, for $u \in K$

$$
\lambda \int_{0}^{1} H(t, s) b(v) g(u(v)) \leq \lambda \int_{0}^{1} \psi(v) b(v) g(u(v)) d v .
$$

Let

$$
M=\max \left\{f(u): 0 \leq u \leq N \lambda \int_{0}^{1} \psi(v) b(v) d v\right\}
$$

and let

$$
R_{3}>\max \left\{2 R_{2}, M \lambda \int_{0}^{1} \psi(s) a(s) d s\right\} .
$$

Then, for $u \in K$ with $\|u\|=R_{3}$, we obtain

$$
\begin{aligned}
Q_{\lambda} u(t) & \leq \lambda \int_{0}^{1} \psi(s) a(s) M d s \\
& \leq R_{3}=\|u\|,
\end{aligned}
$$

so that $\left\|Q_{\lambda} u\right\| \leq\|u\|$. If we set $\Omega_{2}=\left\{u \in B:\|u\| \leq R_{3}\right\}$, then, for $u \in K \cap \partial \Omega_{2}$,

$$
\left\|Q_{\lambda} u\right\| \leq\|u\|, \quad u \in K \cap \partial \Omega_{2} .
$$

Case(ii). $g$ is unbounded, there exists $R_{3}>\max \left\{2 R_{2}, \bar{R}_{1}\right\}$ such that $g(u) \leq g\left(R_{3}\right)$, for $0<u \leq R_{3}$ Similarly, there exists $R_{4}>$ $\max \left\{R_{3}, \lambda \int_{0}^{1} \psi(v) b(v) g\left(R_{3}\right) d v\right\}$ such that $f(u) \leq f\left(R_{4}\right)$, for $0<u \leq R_{4}$.

Let $u \in K$ with $\|u\|=R_{4}$, from $\left(H_{4}\right)$, we have

$$
\begin{aligned}
Q_{\lambda} u(t) & \leq \lambda \int_{0}^{1} \psi(s) a(s) f\left(\lambda \int_{0}^{1} \psi(v) b(v) g\left(R_{3}\right) d v\right) d s \\
& \leq \lambda \int_{0}^{1} \psi(v) a(v) f\left(R_{4}\right) d v \\
& \leq \lambda \int_{0}^{1} \psi(v) a(v)\left(f_{\infty}+\varepsilon\right) R_{4} d v \\
& \leq R_{4}=\|u\| .
\end{aligned}
$$

So, $\left\|Q_{\lambda} u\right\| \leq\|u\|$. If we set $\Omega_{2}=\left\{u \in C[0,1] \mid\|u\|<R_{4}\right\}$, then

$$
\left\|Q_{\lambda} u\right\| \leq\|u\|, \quad \text { for } \quad u \in K \cap \partial \Omega_{2} .
$$

Thus, in either of cases, From of part (ii) of Theorem 2.4, the operator $Q_{\lambda}$ has a fixed point $u^{*} \in K \cap\left(\bar{\Omega}_{2} / \Omega_{1}\right)$. This means that the BVPs (1.1)-(1.2) has a nonnegative solution $(u, v)$ for the chosen value of $\lambda$. The proof is complete. 


\section{Examples}

Consider the nonlinear differential equations with parameter $\lambda$,

$$
\begin{cases}\left.u^{(4)}(t)\right)=\lambda t v(t)\left(v(t) e^{-v(t)}+\frac{v(t)+K}{1+\eta v(t)}\right), & 0<t<1, \\ v^{(4)}(t)=\lambda t u(t)\left(u(t) e^{-u(t)}+\frac{u(t)+K}{1+\eta u(t)}\right), & 0<t<1,\end{cases}
$$

subject to two-point boundary conditions

$$
\left\{\begin{array}{l}
u(0)=0, u^{\prime}(0)=0, u^{\prime \prime}(1)=0, u^{\prime \prime \prime}(1)=0 \\
v(0)=0, v^{\prime}(0)=0, v^{\prime \prime}(1)=0, v^{\prime \prime \prime}(1)=0
\end{array}\right.
$$

Where $a(t)=b(t)=t, f(v)=v\left(v e^{-v}+\frac{v+K}{1+\eta v}\right)=, g(u)=u\left(1+\frac{u+K}{1+\eta u}\right)$. By simple calculations, we have $g(0)=0, f_{\infty}=g_{\infty}=\frac{1}{\eta}, f_{0}=$ $g_{0}=K$. Choosing $\theta=\frac{1}{3}, \eta=100$, and $K=10^{4}$, we obtain $L_{3} \cong 1,1817237, L_{4} \cong 9,1666667$.

By Theorem 3.2 it follow that for every $\lambda$ such that $1,1817237<\lambda<9,1666667$, the BVPs (4.1)-(4.2) has a nonnegative solution $(u, v)$ for given $\lambda$.

\section{References}

[1] R. B. Agarwal, D. O’Regan, P. J. Wang, Positive solutions of differential, Difference, and integral equations, Kluwer Academic, Boston, Ma, 1999.

[2] D. R. Anderson, R. I. Avery, A fourth-order four-point right focal boundary value problem, Rocky Mountain J. Math., 36 (2006), $367-380$.

[3] N. Bouteraa, S. Benaicha, Triple positive solutions of higher-order nonlinear boundary value problems, J. Comput. Sci. Comput. Math., 7 (2017), 25-31.

[4] N. Bouteraa, S. Benaicha, Nonlinear boundary value problems for higher-order ordinary differential equation at resonance, Romanian J. Math. Comput. Sci., 2 (2018), 83-91.

[5] N. Bouteraa, S. Benaicha, Existence of solutions for third-order three-point boundary value problem, Mathematica, 60(83) (2018), 12-22.

[6] A. Cabada, S. Heikkila, Uniqueness, comparison and existence results for third-order functional initial-boundary value problems, Comput. Math. Appl., 41 (2001), 607-618.

[7] A. Cabada, S. Tersian, Existence and multiplicity of solutions to boundary value problems for fourth-order impulsive differential equations, Bound. Value Probl., 2014, 2014:105.

[8] J. Davis, J. Henderson, Uniqueness implies existence for fourth-order Lidstone boundary value problems, Panamer. Math. J., 8 (1998), 23-35.

[9] H. Djourdem, S. Benaicha, Existence of positive solutions for a nonlinear three-point boundary value problem with integral boundary conditions, Acta Math. Univ. Comenianae, 87 (2018), 167-177.

[10] L. H. Erbe, H. Wang, On -the existence of positive solutions of ordinary differential equations, Proc. Amer. Math. Soc. 120 (1994) $743-748$.

[11] C. P. Gupta, Existence and uniqueness theorem for the bending of an elastics beam equation, Appl. Anal., 26 (1988), $289-304$.

[12] G. Infante, P. Pientramala, A cantilever equation witht nonlinear boundary conditions, Electron. J. Qual. Theory Differe. Equ., Spec. Ed. I., 15 (2009), $1-14$.

[13] R. Ma, L. Xu, Existence of positive solutions for a nonlinear fourth-order boundary value problem, Appl. Math. Lett. 23 (2010), $537-543$.

[14] Y. Sun, C. Zhu, Existence of positive solutions for singular fourth-order three-point boundary value problems, Adv. Differ. Equ., $2013,2013: 51$. doi:10.1186-1847-2013-51.

[15] A. R. Aftabizadeh, Existence and uniqueness theorems for fourth-order boundary value problems, J. Math. Anal. Appl. 116 (1986), 415-426.

[16] E. Alves, T.F.Ma., M.L. Policer, Monotone positive solutions for a fourth order equation with nonlinear boundary conditions value, Nonlinear Anal. 71 (2009), 3834-3841.

[17] C. P. Gupta, Existence and uniqueness theorem for the bending of an elastics beam equation at resonance, J. Math. Anal Appl. 135 (1988), 208-225.

[18] R. Ma, B. Thompson, Nodal solutions for a nonlinear fourth-order eigenvalue problem, Acta Math. Sin. Engl. Ser. 24 (2008), 27-34.

[19] Q. Yao, Local existence of multiple positive solutions to a singular cantiliver beam equation, J. Math. Anal. Appl. 363 (2010), $138-154$.

[20] N. Bouteraa, S. Benaicha, Positive periodic solutions for a class of fourth-order nonlinear differential equations, Numerical Analysis and Applications., 22(1) (2019), 1-14.

[21] N. Bouteraa, S. Benaicha, H. Djourdem, M.E. Benattia, Positive solutions for fourth-order two-point boundary value problem with a parameter, Romanian J. Math. Comput. Sci., 8 (2018), 17-30.

[22] H. Djourdem, S. Benaicha, N. Bouteraa, Existence and iteration of monotone positive solution for a fourth-order nonlinear boundary value problem, Fundam. J. Math. Appl., 1 (2018), 205-211.

[23] L. Hu, L. L. Wang, Multiple positive solutions of boundary value problems for systems of nonlinear second order differential equation, J. Math. Anal. Appl., 355 (2007), 1052-1060.

[24] B. Liu, L. Liu, Y. Wu, Positive solutions for singular systems of three-point boundary value problems, Computers Math. Appl., 53 (2007), 1429-1438.

[25] Y. Zhou, Y. Xu, Positive solutions of three boundary value problems for systems of nonlinear second order ordinary differential equation, J. Math. Anal. Appl., 320 (2006), 578-590.

[26] M. A. Krasnosel'skii, Positive solutions of operator equations, Noordhoff, Groningen, 1964. 